\title{
Editorial: intelligent manufacturing systems towards industry 4.0 era
}

\author{
Ahmad Barari $^{1} \cdot$ Marcos de Sales Guerra Tsuzuki $^{2} \cdot$ Yuval Cohen $^{3} \cdot$ Marco Macchi $^{4}$ \\ Accepted: 25 March 2021 / Published online: 7 April 2021 \\ (c) The Author(s), under exclusive licence to Springer Science+Business Media, LLC, part of Springer Nature 2021
}

Keywords Manufacturing digitalisation · Industry 4.0 $\cdot$ Smart factory $\cdot$ Intelligent manufacturing $\cdot$ Smart manufacturing $\cdot$ Cyber-physical production systems $\cdot$ Internet of things $\cdot$ Digital twin $\cdot$ Artificial intelligence $\cdot$ Additive manufacturing

\section{Introduction}

Intelligent Manufacturing is a key concept, developed during the past decades and nowadays gaining further momentum thanks to the potential brought by the Industry 4.0 vision. The expectation for the future is a transition towards Smart Factories built on the presence of Cyber-Physical Systems (CPS), also designated as Cyber-Physical Production Systems (shortly CPPS) or other similar terms. Trends and issues emerging along this transition are relevant for the future research, whereas the evolution of technologies determines the transformation of the manufacturing enterprises. With the currently observed changes, new scientific directions are discussed towards a smart manufacturing practice.

(Kusiak, 2018) reflects on the future trends in smart manufacturing by means of ten conjectures. Amongst others,

Yuval Cohen

yuvalc@afeka.ac.il

Ahmad Barari

ahmad.barari@uoit.ca

Marcos de Sales Guerra Tsuzuki

mtsuzuki@usp.br

Marco Macchi

marco.macchi@polimi.it

1 Department of Mechanical and Manufacturing Engineering, University of Ontario Institute of Technology, Oshawa, ON L1H 7K4, Canada

2 Escola Politécnica From Universidade de São Paulo, Av. Prof. Mello Moraes, 2231, São Paulo - SP 05508-030, Brazil

3 Department of Industrial Engineering, Tel-Aviv Afeka College of Engineering, Mivtza kadesh St. 38, Tel Aviv, Israel

4 Department of Management, Economics and Industrial Engineering, Politecnico Di Milano, Piazza Leonardo da Vinci, 32, 20133 Milano, Italy we cite: manufacturing digitalisation; the reliance on datadriven modelling; the innovations due to the development of materials, processes and products. (Thoben et al., 2017) remarks the potential of CPS from product design through production and logistics up to maintenance and other activities such as, e.g., recycling; this enables Smart Factories to fulfil dynamic customer demands and integrate humans and automation, whereas new business models are also favoured. (Tao et al., 2018) envisions the role of big data in promising applications for the future of manufacturing and, in the directions for the coming years, indicates Digital Twin technologies to join data and virtual (i.e. digital) models synchronized with the current physical products/assets. (Panetto et al., 2019) presents a vision of the challenges for the future as discussed by the research community of International Federation of Automatic Control (IFAC) Coordinating Committee 5, today named Cyber-Physical Manufacturing Enterprises. Amongst the challenges, we cite the evolution of manufacturing plant control based on CPPS and the biological transformation in manufacturing, as well as the new control theories built on collaboration and autonomy with self-X capabilities available in the manufacturing shop-floor (self-awareness, self-reconfiguration, etc.).

Overall, Intelligent Manufacturing is a cornerstone of this evolution, essential to fulfil Industry 4.0 vision and a smart manufacturing practice. Therefore, it is worth developing a Special Issue in this Journal in order to provide a contribution to the development of Intelligent Manufacturing Systems (IMS) along this digital era. The Special Issue was in fact conceptualized during the 13th IFAC workshop on IMS, organized in Oshawa, Ontario, Canada, in August 2019. This Journal was seen as the natural context where the Special Issue could be provided, in light of the community interested in Intelligent Manufacturing at large and considering the establishment of this Journal in 1990 marking a key step in the historical development of the Intelligent Manufacturing concept. 
This Editorial provides a short historical and future perspective on Intelligent and Smart Manufacturing, underlining the main characteristics of the two concepts. Later, we introduce a summary of the evidences available from the selected papers published in this Special Issue, also proposing a categorization of application areas for Intelligent Manufacturing. We conclude with a reflection on the commonalities and differences between the two concepts of Intelligent Manufacturing and Smart Manufacturing, envisioning future directions in the transition we are living towards a full establishment of the Industry 4.0 era.

\section{History and future of intelligent and smart manufacturing}

This Special Issue has origin in the IMS working group, a scientific community developed consistently with the historical move of Intelligent Manufacturing and affiliated to the IFAC Technical Committee 5.1, namely TC 5.1 Manufacturing Plant Control. IMS working group is the promoter of the series of IFAC international IMS workshops. First held in Dearborn, USA, in 1992, the workshop then moved in different countries worldwide-Austria, Romania, Korea, Brazil, Poland, Hungary, Spain, Poland, Portugal, Brazil, USA, and Canada. The 14th IMS 2022 is already scheduled in Israel.

Smart Manufacturing was coined few years after Intelligent Manufacturing, and is nowadays highly used in conjunction with Industry 4.0 in order to indicate a modern manufacturing influenced by the current key enabling technologies and the organizational development that is induced so far. NIST and its definitions of Smart Manufacturing (Lu et al., 2016) are a well-known reference, in line with the evidence that USA is a dominant country in the adoption of the term.

Although the objectives and methodologies are similar, there are evidences of different ways Smart Manufacturing and Intelligent Manufacturing are addressed in the literature (Mittal et al, 2019; Muhuri et al. 2019; Wang et al., 2020).

Smart Manufacturing deals with a set-level of inborne, intrinsic, and agile cleverness that supports quick responses to the needs of all components in the supply chain network, with a common focus on quality, productivity, and sustainability, using a wide range of digitization methods of Industry 4.0. The methods and systems that are utilized more intensively by Smart Manufacturing are those related to Internet of Things, cloud computing, (big) data analytics, and are adopted in different control processes of manufacturing operations including schedule planning, diagnosis and assessment, predictive maintenance, and quality. The Smart Manufacturing systems are also well known for their capabilities to deal with large volumes of unstructured data, and promote decentralization with the use of distributed systems.
Intelligent Manufacturing addresses creative and progressive levels of cleverness which supports its own growth, its understand-ability as a manufacturing system, and the adaptation to the dynamic environment without compromising objectives. Traditionally, and considering the research in the past three decades, the IMS mostly try to seek for the solution using their strong knowledge-based mechanisms. The developed IMS are usually aimed to eliminate the needs to even highly skilled humans and, in case of including highly skilled humans, they assume them in a loop of human-cyberphysical systems. IMS have their manifestations in different application fields; it leads to correspondent terms such as Intelligent products, Intelligent assets, Intelligent services, Intelligent maintenance systems and alike.

We assume that the future requirements in manufacturing will consistently need further developments in both paradigms of Intelligent Manufacturing and Smart Manufacturing, in interaction with each other, confirming the role of Intelligence and Intelligent Manufacturing Systems as a key element of a Smart Factory. Indeed, Intelligent Manufacturing will not be detached from the current development of Industry 4.0 and should be considered as an engine generating the cleverness embedded in the smart manufacturing practice.

\section{Summary of the evidences brought by the selected papers}

Four areas, representative of the application scenarios of Intelligent Manufacturing, are addressed by the papers selected for this Special Issue. The four application areas are hereafter defined.

1. Intelligent Design and Manufacturing deals with intelligent product design with consideration of the manufacturing and production limitations, and intelligent manufacturing technologies to address the needs of advanced product development. The research in this area integrates the upstream activities of product design, process planning, plant design and commissioning with the downstream activities of manufacturing processes and operations in an intelligent platform.

2. Intelligent Process and Production Control considers an intelligent production management practice built on monitoring and control of production processes, production plans and shop-floor schedules, with the end purpose to dynamically optimize the process and planning parameters while being field-synchronized with the manufacturing execution.

3. Intelligent Quality Inspection, Monitoring and Control considers an intelligent quality management practice built on inspection, monitoring and control of the prod- 
uct quality through an integral approach that includes a range of activities such as intelligent inspection processes and procedures to generate the data, data analytics and data processing to interpret information related to quality of the product, monitoring the product quality though the obtained data and information, detection of quality defects and production quality control, early warning of quality defects, rapid diagnosis of root causes, and quality prediction.

4. Intelligent Maintenance regards an intelligent maintenance management practice built on performance-driven decision support, the full integration of maintenance within operations and business management (by means of a cross-functional integration with production, logistics, quality, Health, Safety \& Environment, finance, etc.), and Prognostics and Health Management.

The evidences presented by the selected papers show a variety of methodologies and technologies that are part of smart manufacturing practice. The reliance on advanced analytics, modelling and simulation is the first and major evidence. Data-driven modelling is adopted in different papers across the four application areas; it consists of various methods and algorithms developed from Artificial Intelligence (Machine learning, Deep learning) and from statistics (Statistical modelling). Furthermore, the Digital Twins are emergent technologies leading to a joined use of data and virtual (digital) models; simulation is a key element to build virtual models, and is present in different typologies (Monte Carlo Simulation, Discrete Event Simulation and even System Dynamics). As a second evidence, it is worth underlining that a couple of papers address innovative solutions for more reliable and intelligent manufacturing technology and processes. Overall, this Special Issue provides a balanced distribution of contributions across the application areas, namely five papers in "Intelligent Design and Manufacturing", five papers in "Intelligent Process and Production Control", two papers in "Intelligent Quality Inspection, Monitoring and Control", and three papers in "Intelligent Maintenance".

\section{Conclusions}

The digital transformation induced by Industry 4.0 and its embedded Artificial Intelligence pave the way towards Smart Factories built on the presence of CPPS. Modern shop-floors nowadays experience the confluence of many novel digital technologies that when applied skilfully are designated as Smart Manufacturing. Nevertheless, we are confident that Intelligent manufacturing is sometimes used as a synonym of Smart Manufacturing (see e.g. Thoben et al., 2017). Indeed, we understand that Intelligent Manufacturing can be considered as a concept nowadays embedded within the smart manufacturing practice. The two concepts are respectively sharing various common features. For example:

- Both intelligent and smart manufacturing have advanced (clever) digital support for decision making.

- Both intelligent and smart manufacturing claims the inclusion of key enabling technologies.

- Both intelligent and smart manufacturing rely on modelling approaches to support the decisions with (intelligent/smart) data and models (while this has been related in past decades to Intelligent Manufacturing, now the discussion continues within the Smart Manufacturing arena).

Smart Manufacturing has broadened the scope of Intelligent Manufacturing due to recently developed key features of the digital era, or the novel perspectives recently developed in the scientific and technical community. For example:

- Smart Manufacturing takes advantage of the current development of the key enabling technologies such as, amongst others, augmented reality, additive manufacturing, blockchain, computer vision, and the big data analytics adopted for a data-driven approach, or the incorporation of new technologies for data storage and processing, such as fog and edge computing (Tao et al., 2018).

- Smart Manufacturing emphasizes the connection between physical and cyber layer. It is pushing to a reconsideration of the purpose of control/automation on the shop-floor, leading to innovative self-X capabilities as well as Digital Twins technologies equipping the CPPS with the characteristic of virtualization. Improved capabilities are then available, within or besides the shop-floor, to process data to extract information and to support informed decisions in real-time (Panetto et al., 2019).

- Smart Manufacturing includes organizational and human aspects related to the scientific design of production systems and production processes in a human-centric perspective using advanced technologies (Fantini et al., 2020) and, in particular, collaborative technologies. A relevant focus regards also the way roles are changing with the technologies brought by Industry 4.0, and the skills required in the future, see e.g. (Romero et al., 2016) with the vision of the "Operator 4.0".

In each and every case related to smart manufacturing, Intelligence (or Intelligent Manufacturing) is present (either at the background, or at the front) to answer the requirements of a smart manufacturing practice. Therefore, we consider the outcome of this Special Issue a repository that helps to confirm the role of Intelligence and Intelligent Manufacturing Systems as a key element of a Smart Factory, while 
contributing to the discussion about the scientific directions to a full achievement of the Industry 4.0 potential. Concluding, we believe that three main trends are expected as future scientific discussions.

- The smart manufacturing practice will reinforce the key role of intelligence to support decisions throughout different domains. This will allow to fully reap the benefits of digitalisation, in different terms: (i) an effective and efficient control of the operations in the manufacturing shop-floor, (ii) a closer integration of the design and process planning of the manufactured products with the manufacturing stage; (iii) an integrated approach across different domains required to manage the manufacturing operations (production, quality, maintenance, logistics, safety, etc.).

- The cyber-physical integration will be an essential technological "platform" nurturing intelligence, and facilitating the exploitation of advanced analytics, modelling, simulation, and optimization. The cyber-physical integration will also provide a key "platform" for the manufacturing technologies (such as additive manufacturing) and the advanced automation solutions (as collaborative robots, mobile robots, autonomous vehicles and drones, etc.).

- Intelligence will continue to be a core required element for solving problems and controlling the shop-floor, and this will be influenced by the trends of sustainability and circular economy. Intelligent decision-making, and intelligent criteria and solutions, are also essential for solving big issues, inherently requiring to act on multiple parameters across different domains (products, materials, processes, systems, etc.) and under a long-term perspective.

\section{References}

Fantini, P., Pinzone, M., \& Taisch, M. (2020). Placing the operator at the centre of Industry 4.0 design: Modelling and assessing human activities within cyber-physical systems. Computers and Industrial Engineering, 139, 105058. https://doi.org/10.1016/j.cie.2018. 01.025 .

Kusiak, A. (2018). Smart manufacturing. International Journal of Production Research, 56(1-2), 508-517. https://doi.org/10.1080/ 00207543.2017.1351644.

Lu, Y., Morris, K., \& Frechette, S. (2016). Current standards landscape for smart manufacturing systems. NIST Interagency/Internal Report (NISTIR), National Institute of Standards and Technology, Gaithersburg, MD. Retrieved March 20, 2021, from https://doi. org/10.6028/NIST.IR.8107

Mittal, S., Khan, M. A., Romero, D., \& Wuest, T. (2019). Smart manufacturing: Characteristics, technologies and enabling factors. Proceedings of the Institution of Mechanical Engineers, Part B: Journal of Engineering Manufacture, 233(5), 1342-1361. https:// doi.org/10.1177/0954405417736547.

Muhuri, P., Shukla, A., \& Abraham, A. (2019). Industry 4.0: A bibliometric analysis and detailed overview. Engineering Applications of Artificial Intelligence, 78, 218-235. https://doi.org/10.1016/j. engappai.2018.11.007.

Panetto, H., Iung, B., Ivanov, D., Weichhart, G., \& Wang, X. (2019). Challenges for the cyber-physical manufacturing enterprises of the future. Annual Reviews in Control, 47, 200-213. https://doi. org/10.1016/j.arcontrol.2019.02.002.

Romero, D., Bernus, P., Noran, O., Stahre, J., \& Fast-Berglund, Å. (2016). The operator 40: Human cyber-physical systems and adaptive automation towards human-automation symbiosis work systems. In I. Nääs, O. Vendrametto, J. M. Reis, R. F. Goncalves, M. T. Silva, G. von Cieminski, \& D. Kiritsis (Eds.), Advances in production management systems initiatives for a sustainable world APMS 2016 IFIP advances in information and communication technology. (Vol. 488, pp. 677-686). Springer.

Tao, F., Qi, Q., Liu, A., \& Kusiak, A. (2018). Data-driven smart manufacturing. Journal of Manufacturing Systems, 48(Part C), 157-169. https://doi.org/10.1016/j.jmsy.2018.01.006.

Thoben, K.-D., Wiesner, S., \& Wuest, T. (2017). "Industrie 4.0" and smart manufacturing-A review of research issues and application examples. International Journal of Automation Technology, 11(1), 4-16. https://doi.org/10.20965/ijat.2017.p0004.

Wang, B., Tao, F., Fang, X., Liu, C., Liu, Y., \& Freiheit, T. (2020). Smart manufacturing and intelligent manufacturing: A comparative review. Engineering. https://doi.org/10.1016/j.eng.2020.07. 017 In-press.

Publisher's Note Springer Nature remains neutral with regard to jurisdictional claims in published maps and institutional affiliations. 